MIT-CTP-3519

\title{
Symmetries of Discontinuous Flows and the Dual Rankine-Hugoniot Conditions in Fluid Dynamics
}

\author{
Oliver Jahn ${ }^{1}$ \\ Center for Theoretical Physics \\ Massachusetts Institute of Technology \\ Cambridge, MA 02139-4307 \\ U.S.A. \\ V. V. Sreedhar ${ }^{2}$ \\ Department of Physics \\ Indian Institute of Technology \\ Kanpur, 208016 \\ India \\ Amitabh Virmani ${ }^{3}$ \\ Department of Physics \\ University of California \\ Santa Barbara, CA 93106-9530 \\ U.S.A.
}

\begin{abstract}
It has recently been shown that the maximal kinematical invariance group of polytropic fluids, for smooth subsonic flows, is the semidirect product of $S L(2, R)$ and the static Galilei group $G$. This result purports to offer a theoretical explanation for an intriguing similarity, that was recently observed, between a supernova explosion and a plasma implosion. In this paper we extend this result to discuss the symmetries of discontinuous flows, which further validates the explanation by taking into account shock waves, which are the driving force behind both the explosion and implosion. This is accomplished by constructing a new set of RankineHugoniot conditions, which follow from Noether's conservation laws. The new set is dual to the standard Rankine-Hugoniot conditions and is related to them through the $S L(2, R)$ transformations. The entropy condition, that the shock needs to satisfy for physical reasons, is also seen to remain invariant under the transformations.
\end{abstract}

\footnotetext{
1 jahn@mit.edu

${ }^{2}$ sreedhar@iitk.ac.in

3 virmani@physics.ucsb.edu
} 


\section{Introduction}

It has recently been observed that the density profiles of a supernova explosion and an inertial confinement plasma implosion 1, 2, 3] are strikingly similar. An empirical basis for this intriguing duality between explosion and implosion was given by Drury and Mendonça [4 who pointed out that Euler's equations of fluid dynamics, which describe both the systems, are form-invariant under a set of nonlinear coordinate transformations viz. $\vec{x} \rightarrow \vec{x} / t, \quad t \rightarrow-1 / t$. The minus sign in the time transformation maps an explosion to an implosion and the inversion allows large time scales to be mapped to small time scales and vice versa. These transformations suggest that the maximal kinematical invariance group $\mathcal{G}$ of fluid dynamics is larger than the standard Galilei group. It is now known that this larger group is a twelve-parameter semidirect product, $\mathcal{G}=S L(2, R) \wedge G$ [5], 6], where $G$ is the nine-parameter, connected, static Galilei group:

$$
\vec{x} \rightarrow \mathbf{R} \vec{x}+\vec{v} t+\vec{a}, \quad t \rightarrow t
$$

and $S L(2, R)$ is the group consisting of the transformations:

$$
t \rightarrow \frac{\alpha t+\beta}{\gamma t+\delta}, \quad \vec{x} \rightarrow \frac{\vec{x}}{\gamma t+\delta} \quad \text { with } \quad \alpha \delta-\beta \gamma=1 .
$$

Physically, the three-parameter $S L(2, R)$ group consists of time translations, scale transformations, and a one-parameter set of time-dependent scale transformations called expansions. The transformations proposed by Drury and Mendonça are a special case of the $S L(2, R)$ transformations with $(\alpha, \beta, \gamma, \delta)=$ $(0,-1,1,0)$. The $S L(2, R)$ part of $\mathcal{G}$ is therefore important for a better understanding of the explosion-implosion map.

It should be pointed out that the naive expectation of using time-reversal invariance, to explain the similarity between explosion and implosion, is untenable here since the length and time scales involved in the two systems are drastically different. Invoking scaling arguments is not of much help since, although a composition of time-reversal and suitable scalings leaves the equations of fluid mechanics and the Reynolds number invariant, it has the property of reversing the direction of time's arrow and thereby violates the second law of thermodynamics. As a consequence, when applied to a shock wave, such transformations violate entropy conditions that define the physicality of the shock. As is wellknown, however, both the supernova explosion and the plasma implosion are driven by the formation and propagation of a shock wave. It is therefore important to examine whether the $S L(2, R)$ symmetry, that purports to explain the observed duality, respects the physicality of the shock wave. With this in view, we extend the study of [5] - in which the explanation of explosion-implosion duality based on the symmetry group $\mathcal{G}$ was restricted to smooth, subsonic flows - to examine shock waves. It will be shown that shock wave solutions are consistent with the symmetries of the maximal kinematical invariance group $\mathcal{G}$ in the following precise sense.

A shock in a fluid is described mathematically by the well-known RankineHugoniot jump conditions [7]. So the natural question to ask is: What happens 
to these conditions under the action of the $S L(2, R)$ group? This question is best answered not in the framework of the partial differential equations of fluid dynamics, but by reverting back to their so-called primitive form i.e. expressing them as conservation laws. The conservation laws are completely equivalent to the partial differential equations for smooth flows, but produce the RankineHugoniot jump conditions for discontinuous flows in a natural and well-defined manner. The connection with $S L(2, R)$ is made by appealing to Noether's theorem which asserts that corresponding to every continuous symmetry, there exists a conserved charge.

Anticipating that the $S L(2, R)$ transformations will mix the conservation laws corresponding to various symmetries, we construct the Noether charges corresponding to them and the boost transformations, in addition to the wellknown ones for rotations, space and time translations. We then use the attendant conservation laws to establish a new set of jump conditions. It turns out that the new conditions are identically satisfied if the standard conditions for mass, momentum and energy conservation are satisfied. Although seemingly redundant because of this reason, the new set holds independently; following, as it does, from the symmetries of the fluid equations. In fact, these conditions are useful to prove the form-invariance of the Rankine-Hugoniot conditions under the $S L(2, R)$ transformations. Thus, to each physical system governed by the fluid dynamics equations two independent, but physically equivalent, sets of jump conditions can be associated, the two being related by $S L(2, R)$ transformations. We conclude that the $S L(2, R)$ transformations map the RankineHugoniot conditions of the explosion to the dual Rankine-Hugoniot conditions of the implosion and vice versa. Further, by specialising to the Drury-Mendonça transformations, $\vec{x} \rightarrow \vec{x} / t, \quad t \rightarrow-1 / t$, we show that the jump conditions for boosts and expansions, along with the continuity equation for mass conservation, provide an independent, albeit equivalent, description of the shock. They may be viewed either as the dual of the standard Rankine-Hugoniot conditions, or, in the language of passive coordinate transformations, as the standard RankineHugoniot conditions in the dual coordinate system corresponding to the choice $(\alpha, \beta, \gamma, \delta)=(0,-1,1,0)$. Similar dual conditions exist for each choice of the $S L(2, R)$ parameters.

It is well-known that Rankine-Hugoniot conditions describe not only shocks, but other discontinuities like slip and contact discontinuities, for example. Therefore, the map between the dual sets of Rankine-Hugoniot conditions would be relevant to explosion-implosion duality only if both the sets refer to shocks. In other words, only those Rankine-Hugoniot conditions that describe a shock and only those $S L(2, R)$ transformations which map a shock to a shock are of interest for explosion-implosion duality. Moreover, Rankine-Hugoniot conditions say nothing about the physicality of the shock - this information is contained in additional inequalities for its entropy that the shock needs to satisfy. Physical shocks are distinguished from others because their entropy always increases across the shock front. We verify explicitly that this requirement is unaffected by the $S L(2, R)$ transformations.

The physicality of the map between explosion and implosion may also be es- 
tablished in the following subtle manner: Although the notions of viscosity and heat conduction lose their meaning in the immediate vicinity of the shock, because the changes in all the quantities they depend on are so great, they do play an important role in the formation and maintenance of a shock discontinuity [8]. In particular, the positivity of the coefficients of viscosity and heat conduction guarantees that the shock satisfies the appropriate entropy conditions [8] Hence, Euler's equations ought to be considered as a special case of a more general set of fluid equations with vanishingly small viscosity. Requiring the sign of the viscosity to remain unchanged under the transformations establishes the physicality. The Navier-Stokes equations - which are the obvious choice for including viscosity - are not invariant under the full $S L(2, R)$ part of $\mathcal{G}$, but only under the standard Galilean transformations. However, a more general set of fluid equations with viscosity fields transforming appropriately under the $S L(2, R)$ transformations has a maximal kinematical invariance group given by $\mathcal{G}[5]$. Hence we use these equations for our purpose of examining the behaviour of non-vanishing viscosity under the $S L(2, R)$ transformations. Similar arguments apply for heat conduction, but it does not bring in any new qualitative features and hence is omitted from further discussion.

\section{Symmetries of Fluid Dynamics}

In this section we briefly recapitulate the results of [5]. The general fluid equations in $n$-dimensional space are [9]

$$
\begin{aligned}
D \rho & =-\rho \vec{\nabla} \cdot \vec{u} \\
\rho D \vec{u} & =-\vec{\nabla} p+\vec{V} \\
D \varepsilon & =-(\varepsilon+p) \vec{\nabla} \cdot \vec{u}
\end{aligned}
$$

where

$$
D=\frac{\partial}{\partial t}+\vec{u} \cdot \vec{\nabla}
$$

and

$$
V_{i}=\nabla_{j}\left(\eta\left(\nabla_{j} u_{i}+\nabla_{i} u_{j}-\frac{2}{n} \delta_{i j} \nabla_{k} u_{k}\right)\right)+\nabla_{i}\left(\zeta \nabla_{k} u_{k}\right)
$$

In the above equations $\rho, \vec{u}, p, \varepsilon$ stand for the density, velocity, pressure and energy density of the fluid respectively and $\eta, \zeta$ are the bulk and shear viscosity fields.

The above differential equations are usually augmented by an algebraic condition called the polytropic equation of state which relates the pressure to the energy density as

$$
p=\left(\gamma_{0}-1\right) \varepsilon
$$

where $\gamma_{0}$ is a constant called the polytropic exponent. As shown in [5], the maximal invariance group of the above set of equations is $\mathcal{G}=S L(2, R) \wedge G$, 
provided the polytropic exponent takes the standard value for an ideal, nonrelativistic fluid viz. $\gamma_{0}=1+\frac{2}{n}$. For this value, the fluid equations are invariant under the following transformations [5]:

\section{Connected, static Galilei transformations:}

Let $g$ denote a general element of this sub-group then

$$
g: \quad t^{\prime}=t, \quad \vec{x}^{\prime}=\mathbf{R} \vec{x}+\vec{v} t+\vec{a}
$$

with $\mathbf{R}$ an orthogonal matrix. Under the action of $g$, the fields $\rho$ and $\vec{u}$ transform as

$$
\rho^{\prime}=\rho \quad \text { and } \quad \vec{u}^{\prime}=\vec{u}+\vec{v}
$$

\section{SL $(2, R)$ transformations:}

Let $\sigma$ denote a general element of the $S L(2, R)$ part of $\mathcal{G}$ then

$$
\sigma: \quad t^{\prime}=\frac{\alpha t+\beta}{\gamma t+\delta}, \quad \vec{x}^{\prime}=\frac{\vec{x}}{\gamma t+\delta} \quad \text { where } \quad \alpha \delta-\beta \gamma=1
$$

Under the action of $\sigma$, the fields transform as

$$
\rho^{\prime}=(\gamma t+\delta)^{n} \rho \quad \text { and } \quad \vec{u}^{\prime}=(\gamma t+\delta) \vec{u}-\gamma \vec{x}
$$

For both $g$ and $\sigma$, the transformations of $\varepsilon$ and $p$ can be worked out once the transformation properties of $\rho$ are known since

$$
\varepsilon=\chi \rho^{\gamma_{0}}, \quad p=\left(\gamma_{0}-1\right) \varepsilon,
$$

with the field $\chi$ - related to entropy - transforming like a scalar. The transformation properties of the viscosity fields are similar to the density $\rho$

$$
\eta^{\prime}=(\gamma t+\delta)^{n} \eta \quad \text { and } \quad \zeta^{\prime}=(\gamma t+\delta)^{n} \zeta
$$

The above results were derived in [5] by requiring the invariance of the Action for the simple case of an inviscid and isentropic fluid. The symmetry of the equations followed by subsequently relaxing the simplifications to arrive at the general fluid equations. It should be noted that the requirement of the invariance of the Action is sufficient, but not necessary, for the form invariance of the equations that follow from it. Any transformation that leaves the Action invariant upto a multiplicative factor produces equations of motion which have the same form. If this is taken into account, the condition $\alpha \delta-\beta \gamma=1$ is no longer required and $S L(2, R)$ gets replaced by $G L(2, R)$ in the maximal invariance group of the general fluid equations. However, it is sufficient for our purposes to concentrate on the variational symmetries of the fluid equations and for this purpose, $\mathcal{G}=S L(2, R) \wedge G$. Finally, for the sake of completeness, it should also be pointed out that the $S L(2, R)$ condition is invariant under the following discrete symmetries $(\alpha, \beta, \gamma, \delta) \rightarrow(\alpha,-\beta,-\gamma, \delta), \rightarrow(-\alpha, \beta, \gamma,-\delta), \rightarrow(-\alpha,-\beta,-\gamma,-\delta)$ of the $S L(2, R)$ parameters. 


\section{Conservation Laws}

In this section we construct the conservation laws corresponding to the symmetries outlined in the previous section. In order to do this, it is useful to revert back to the Action formalism and obtain the results for the subclass of inviscid, isentropic and irrotational flows. The corresponding expressions for a general fluid can then be worked out along the lines of $[5]$.

For inviscid, isentropic and irrotational flows, the Lagrangian density is given by

$$
\mathcal{L}=\rho\left(\dot{\phi}-\frac{1}{2}(\vec{\nabla} \phi)^{2}\right)-\rho^{\gamma_{0}}
$$

where $\vec{\nabla} \phi$ stands for the curl-free part of the velocity vector field $\vec{u}$. Let $\mu=0,1,2,3$ and $x^{\mu}$ be a four-vector under the transformations of the previous section i.e. $x^{i}$ with $i=1,2,3$ are the components of $\vec{x}$ and $x^{0}=t$. Let the infinitesimal variations in the coordinates and fields be defined as

$$
\delta x^{\mu}=x^{\mu^{\prime}}-x^{\mu} \quad \text { and } \quad \delta \phi(x)=\phi^{\prime}\left(x^{\prime}\right)-\phi(x)
$$

Then the variations for translations, rotations, boosts, dilatations, and expansions respectively are given by

$$
\delta x^{\mu}=a^{\mu}, \quad \delta x^{i}=\omega^{i j} x^{j}, \quad \delta x^{i}=v^{i} t, \quad \delta x^{i}=\lambda x^{i}, \quad \delta t=2 \lambda t \quad \text { and } \delta x^{\mu}=-\mu t x^{\mu}
$$

where the parameters $\lambda, a^{0}, \mu$ are expressible in terms of the $S L(2, R)$ parameters $\alpha, \beta, \gamma, \delta$. The field variation is given by

$$
\delta \phi=\Lambda=\frac{[\gamma(x+a)-\delta v]^{2}}{2 \gamma(\gamma t+\delta)}
$$

The variation in $\rho$ is not important since no derivatives of $\rho$ appear in the Lagrangian density. Using these results we find, by a straightforward application of Noether's theorem [10], that the following quantities, integrated over all space, are constants of motion:

Temporal Translations : $\quad H=\frac{\rho}{2}(\vec{\nabla} \phi)^{2}+\rho^{\gamma_{0}}$

$$
\begin{aligned}
& \text { Spatial Translations : } \quad \vec{P}=\rho \vec{\nabla} \phi \\
& \text { Rotations : } \quad \vec{L}=\vec{P} \times \vec{x} \\
& \text { Boosts : } \quad \vec{K}=\vec{P} t-\rho \vec{x}
\end{aligned}
$$


The conditions of irrotationality and isentropicity can be relaxed easily and one sees that Euler's equations

$$
\begin{gathered}
\dot{\rho}=-\vec{\nabla} \cdot(\rho \vec{u}) \\
\rho \dot{\vec{u}}=-\rho(\vec{u} \cdot \vec{\nabla}) \vec{u}-\vec{\nabla} p \\
\dot{\varepsilon}=-\vec{\nabla} \cdot(\varepsilon \vec{u})-p \vec{\nabla} \cdot \vec{u}
\end{gathered}
$$

can be expressed in the form of conservation laws,

$$
\begin{gathered}
\frac{\partial}{\partial t} \rho=-\frac{\partial}{\partial x_{j}}\left(\rho u_{j}\right) \\
\frac{\partial}{\partial t}\left(\rho u_{i}\right)=-\frac{\partial}{\partial x_{j}}\left(\rho u_{i} u_{j}+\delta_{i j} p\right) \\
\frac{\partial}{\partial t}\left(\frac{1}{2} \rho \vec{u}^{2}+\varepsilon\right)=-\frac{\partial}{\partial x_{j}}\left[\left(\frac{1}{2} \rho \vec{u}^{2}+\varepsilon+p\right) u_{j}\right]
\end{gathered}
$$

for mass and the translation generators found above. These can be reexpressed succinctly as follows:

$$
\partial_{\mu} J_{(\rho)}^{\mu}=0, \quad \partial_{\mu} J_{(\vec{P})}^{\mu}=0, \quad \text { and } \quad \partial_{\mu} J_{(H)}^{\mu}=0
$$

The zeroeth components of the above currents, namely $\rho, \rho \vec{u}$, and $\frac{1}{2} \rho \vec{u}^{2}+\varepsilon$, give the charge densities which, when integrated over all space, give the conserved charges. As is well-known, these are merely statements of mass, momentum flux, and total energy conservation. The corresponding current densities are

$$
\begin{gathered}
J_{\rho}^{j}=\rho u_{j} \\
J_{P_{i}}^{j}=\rho u_{i} u_{j}+\delta_{i j} p \\
J_{H}^{j}=\left(\frac{1}{2} \rho \vec{u}^{2}+\varepsilon+p\right) u_{j}
\end{gathered}
$$

The conservation laws corresponding to rotations, boosts, dilatations and expansions can be stated similarly

$$
\partial_{\mu} J_{(\vec{L})}^{\mu}=0, \quad \partial_{\mu} J_{(\vec{K})}^{\mu}=0, \quad \partial_{\mu} J_{(D)}^{\mu}, \quad \text { and } \quad \partial_{\mu} J_{(A)}^{\mu}=0
$$

The charge densities are shown in (19) -(22) respectively, and the corresponding currents are

$$
\begin{gathered}
\vec{J}_{L_{i}}=\epsilon_{i k l} x_{k} \vec{J}_{P_{l}} \\
\vec{J}_{K_{i}}=t \vec{J}_{P_{i}}-x_{i} \vec{J}_{\rho}
\end{gathered}
$$




$$
\begin{gathered}
\vec{J}_{D}=x_{i} \vec{J}_{P_{i}}-2 t \vec{J}_{H} \\
\vec{J}_{A}=\frac{1}{2} \vec{x}^{2} \vec{J}_{\rho}-t x_{i} \vec{J}_{P_{i}}+t^{2} \vec{J}_{H}
\end{gathered}
$$

It may be mentioned that the above results are not surprising in the light of [11, where corresponding results for a free, nonrelativistic, point particle were obtained through a discussion that essentially parallels the above. The noteworthy linear relations between the currents will, however, play a crucial role in this paper when we consider flows with discontinuities.

\section{Discontinuous Flows and Jump Conditions}

As long as the flows are smooth, i.e. the functions $\rho, \vec{u}, p, \varepsilon \in \mathbf{C}^{1}$ in their dependence on $\vec{x}$ and $t$, the systems $(23-25)$ and $(26-28)$ are equivalent. However, real flows are not always smooth and can develop discontinuities as time elapses. Such flows are described by weak solutions of differential equations [7. A weak solution is generally piecewise smooth. The smooth parts satisfy the differential equation in the usual, or strong, form, but that does not generally suffice to determine the course of motion for initial data, and the equation must be supplemented by jump conditions. The resulting jump conditions are most clearly derived from the conservation laws.

By definition any, possibly non-smooth, function $J^{\mu}(\vec{x}, t)$ that satisfies

$$
\int \partial_{\mu} w(\vec{x}, t) J^{\mu}(\vec{x}, t) \mathrm{d}^{3} x \mathrm{~d} t=0
$$

for all test functions $w(\vec{x}, t)$ is said to be a weak solution of the differential equation $\partial_{\mu} J^{\mu}=0$.

We now use the above definition to obtain the jump conditions associated with the system of conservation laws derived in the last section. Suppose $J^{\mu}(\vec{x}, t)$ has a jump discontinuity across a hyper-surface $\mathcal{S}$ in $\vec{x}, t$ space, while otherwise being continuously differentiable in some neighbourhood $\mathcal{N}$ of $\mathcal{S}$ (see Fig.1). Let $w(\vec{x}, t)$ be a test function with support in $\mathcal{N}$. Let $\mathcal{R}$ be the part of the support of $w(\vec{x}, t)$ that lies on one side of $\mathcal{S}$, say the right. Then, by Gauss's theorem

$$
\int_{\mathcal{R}} \partial_{\mu} w J^{\mu} \mathrm{d}^{3} x \mathrm{~d} t+\int_{\mathcal{R}} w \partial_{\mu} J^{\mu} \mathrm{d}^{3} x \mathrm{~d} t=\int_{\mathcal{R}} \partial_{\mu}\left(w J^{\mu}\right) \mathrm{d}^{3} x \mathrm{~d} t=\int_{\mathcal{S}} w n_{\mu} J^{\mu} \mathrm{d}^{3} \mathcal{S}
$$

since $w(\vec{x}, t)=0$ on the boundary of $\mathcal{R}$ except on $\mathcal{S}$. The second integral in the above equation is zero, because the conservation law holds in the strong sense in the interior of $\mathcal{R}$. Here, $n(\vec{x}, t)$ is the outward normal vector to the hypersurface $\mathcal{S}$. Therefore, if we integrate similarly over the left part of the support of $w$, add the result and make use of (38), we find that:

$$
0=\int_{\mathcal{S}} w n_{\mu} \Delta J^{\mu} \mathrm{d} \mathcal{S}
$$




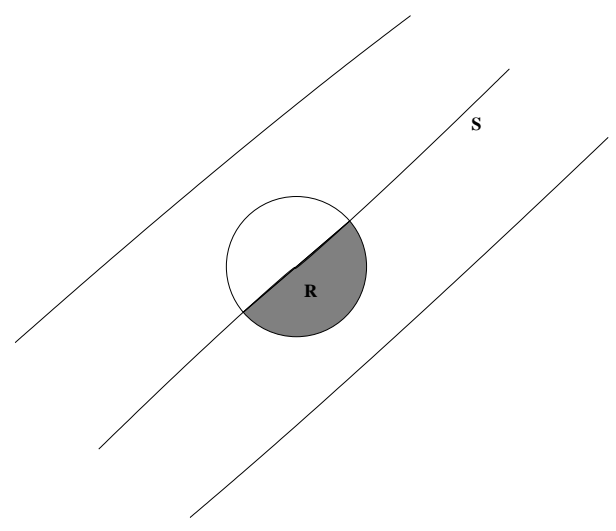

Figure 1: Diagram for the jump condition.

where $\Delta f$ denotes the difference of the two limiting values of a function $f$ on the two sides of the hypersurface $\mathcal{S}$ i.e. the jump of the function. This result follows because the vector $n_{\mu}$, which by convention points outwards, flips its sign on the left side of the support. Since $w$ is an arbitrary test function, the above equation implies the jump condition

$$
n_{\mu} \Delta J^{\mu}=0 \quad \text { on } \mathcal{S} .
$$

Applying (41) to the conservation laws (29) for $J_{(\rho)}^{\mu}, J_{(\vec{P})}^{\mu}$ and $J_{(H)}^{\mu}$, we obtain

$$
\begin{aligned}
& 0=n_{\mu} \Delta J_{(\rho)}^{\mu}, \\
& 0=n_{\mu} \Delta J_{(\vec{P})}^{\mu}, \\
& 0=n_{\mu} \Delta J_{(H)}^{\mu}
\end{aligned}
$$

From here the standard Rankine-Hugoniot conditions can be derived in their usual form 17. Similarly, one can apply (41) to the conservation laws (33) for $J_{(\vec{L})}^{\mu}, J_{(\vec{K})}^{\mu}, J_{(D)}^{\mu}$, and $J_{(A)}^{\mu}$ to obtain a new set of jump conditions:

$$
\begin{aligned}
& 0=n_{\mu} \Delta J_{(\vec{L})}^{\mu} \\
& 0=n_{\mu} \Delta J_{(\vec{K})}^{\mu} \\
& 0=n_{\mu} \Delta J_{(D)}^{\mu} \\
& 0=n_{\mu} \Delta J_{(A)}^{\mu} .
\end{aligned}
$$

Since the coordinates $\vec{x}$ and $t$ are continuous on $\mathcal{S}$, these conditions are all identically satisfied because of the jump conditions for mass, momentum and energy conservation, in (42-44) - a fact that can be easily verified using (19-22) and (34-37). 


\section{The Dual Rankine-Hugoniot Conditions}

We have seen that the new set of jump conditions associated with rotations, boosts, dilatations, and expansions, follow from the jump conditions associated with mass, momentum and energy. This suggests that the Rankine-Hugoniot conditions are invariant under the full kinematical invariance group of smooth flows, including the $S L(2, R)$ part.

To see this explicitly, we consider the transformation properties of the conserved currents under $S L(2, R)$. Let us begin by considering the simplest of these, namely the time-component of $J_{(\rho)}^{\mu}$, i.e. $\rho$. From equation (10), now with $n=3$,

$$
\rho^{\prime}=(\gamma t+\delta)^{3} \rho
$$

The $(\gamma t+\delta)^{3}$ factor is cancelled by an identical factor coming from the change of variables when we perform an integration over all space. Moreover, the transformation does not mix $\rho$ with any other current. Thus, $\rho$ transforms under the singlet representation of $S L(2, R)$ as a scalar density. Let us now consider the transformation of the time-component of $J_{(\vec{P})}^{\mu}$, i.e. $\vec{P}=\rho \vec{u}$. From (10) it now follows, after a little algebra, that

$$
\vec{P}^{\prime}=\rho^{\prime} \vec{u}^{\prime}=(\gamma t+\delta)^{3}(\delta \vec{P}+\gamma \vec{K})
$$

Thus the transformation of the spatial translation generator mixes it with the boost generator together with which, it forms a doublet representation of $S L(2, R)$, with the prefactor $(\gamma t+\delta)^{3}$ now making it a vector density. The latter fact is, in fact, generic to the time-components of all the currents. Likewise, we may consider the generator of time translations, namely the Hamiltonian, and it follows that

$$
H^{\prime}=(\gamma t+\delta)^{3}\left(\gamma^{2} A-\delta \gamma D+\delta^{2} H\right)
$$

Thus the transformation of the time translation generator mixes it with the generator of dilatations and expansions, the three of them form the triplet (or adjoint) representation. The transformation properties of the rest of the currents can be similarly worked out and the results summarised as follows: If the (abstract) symmetry generators $T_{r}$ transform as

$$
T_{r}^{\prime} \equiv \sigma^{-1} T_{r} \sigma=\sum_{s} M_{r s}(\sigma) T_{s}
$$

where the matrix $M(\sigma)$ is determined by the group structure of $S L(2, R) \wedge G$, then the corresponding currents transform as

$$
J_{r}^{\mu \prime}\left(x^{\prime}\right)=\operatorname{det}\left(\frac{\partial x}{\partial x^{\prime}}\right) \frac{\partial x^{\mu \prime}}{\partial x^{\nu}} \sum_{s} M_{r s}(\sigma) J_{s}^{\nu}(x) .
$$


Assembling the currents in a column,

$$
J^{\mu}=\left(\begin{array}{c}
J_{(\rho)}^{\mu} \\
J_{(\vec{K})}^{\mu} \\
J_{(\vec{P})}^{\mu} \\
J_{(A)}^{\mu} \\
J_{(D)}^{\mu} \\
J_{(H)}^{\mu}
\end{array}\right)
$$

one has for the transformation matrix,

$$
M=\left(\begin{array}{cccccc}
1 & 0 & 0 & 0 & 0 & 0 \\
0 & \alpha & \beta & 0 & 0 & 0 \\
0 & \gamma & \delta & 0 & 0 & 0 \\
0 & 0 & 0 & \alpha & -\alpha \beta & \beta^{2} \\
0 & 0 & 0 & -2 \alpha \gamma & (\beta \gamma+\alpha \delta) & -2 \beta \delta \\
0 & 0 & 0 & \gamma^{2} & -\gamma \delta & \delta^{2}
\end{array}\right)
$$

Using $\alpha \delta-\beta \gamma=1$, and the fact that the determinant of a block diagonal matrix is the product of the determinants of the blocks, it is easy to check that the matrix $M$ has unit determinant. As already pointed out, the fact that the currents transform like vector densities is reflected in the temporal components picking up a multiplicative factor $(\gamma t+\delta)^{3}$. The spatial components follow the example

$$
J_{(\rho)}^{i \prime}=(\gamma t+\delta)^{n+1} J_{(\rho)}^{i}-\gamma x^{i}(\gamma t+\delta)^{n} J_{(\rho)}^{0}
$$

with the same $S L(2, R)$ transformations defined by the matrix $M$.

The dual Rankine-Hugoniot conditions are now easily obtained. The normal vector $n_{\mu}$ appearing in the jump condition (41) transforms like a covector,

$$
n_{\mu}^{\prime} \propto \frac{\partial x^{\nu}}{\partial x^{\prime \prime}} n_{\nu},
$$

so the transformed jump condition for $J_{r}$ is

$$
n_{\mu}^{\prime} \Delta J_{r}^{\mu \prime} \propto \operatorname{det}\left(\frac{\partial x}{\partial x^{\prime}}\right) \sum_{s} M_{r s}(\sigma) n_{\mu} \Delta J_{s}^{\mu}(x)=0 \quad \text { on } \mathcal{S} .
$$

Since the determinant is smooth across the surface $\mathcal{S}$, the factor in front of the sum can be omitted. The transformed jump condition is therefore a linear combination of the original jump conditions. In particular, the conditions for $J_{(\rho)}$, $J_{(\vec{P})}$ and $J_{(H)}$ (the Rankine-Hugoniot conditions) become linear combinations of the jump conditions for $J_{(\rho)}, J_{(\vec{P})}, J_{(\vec{K})}, J_{(H)}, J_{(D)}$ and $J_{(A)}$,

$$
\begin{aligned}
n_{\mu}^{\prime} \Delta J_{(\rho)}^{\mu \prime} & \propto n_{\mu} \Delta J_{(\rho)}^{\mu}, \\
n_{\mu}^{\prime} \Delta J_{(\vec{P})}^{\mu \prime} & \propto n_{\mu}\left(\gamma \Delta J_{(\vec{K})}^{\mu}+\delta \Delta J_{(\vec{P})}^{\mu}\right), \\
n_{\mu}^{\prime} \Delta J_{(H)}^{\mu \prime} & \propto n_{\mu}\left(\delta^{2} \Delta J_{(H)}^{\mu}-\gamma \delta \Delta J_{(D)}^{\mu}+\gamma^{2} \Delta J_{(A)}^{\mu}\right) .
\end{aligned}
$$


The standard Rankine-Hugoniot conditions (42-44), in conjunction with the new set of jump conditions (45-48), then imply that the right hand side of the above equations is identically zero i.e. the Rankine-Hugoniot conditions are forminvariant. In particular, this holds for the Drury-Mendonça transformation $t \rightarrow-1 / t, \vec{x} \rightarrow \vec{x} / t$ used to relate the explosion and implosion problems. For this, $(\alpha, \beta, \gamma, \delta)=(0,-1,1,0)$ and it follows that

$$
\begin{aligned}
& 0=n_{\mu} \Delta J_{(\rho)}^{\mu} \\
& 0=n_{\mu} \Delta\left(x_{i} J_{(\rho)}^{\mu}-t J_{\left(P_{i}\right)}^{\mu}\right) \\
& 0=n_{\mu} \Delta\left(-t^{2} J_{(H)}^{\mu}+t x_{i} J_{\left(P_{i}\right)}^{\mu}-\frac{1}{2} x^{2} J_{(\rho)}^{\mu}\right) .
\end{aligned}
$$

where we have substituted the explicit expressions for the currents $J_{(\vec{K})}^{\mu}$ and $J_{(A)}^{\mu}$. The conditions (62-24) are the dual Rankine-Hugoniot conditions. If an explosion is described by the standard Rankine-Hugoniot conditions, the corresponding implosion, obtained by a Drury-Mendonça transformation, is described by the dual Rankine-Hugoniot conditions (62-64).

Since the coordinates $\vec{x}$ and $t$ are continuous on $\mathcal{S}$, and crucially because the relations between the currents are linear, the conditions (62-64) are equivalent to the jump conditions obtained from mass, momentum and energy conservation, in (42-44). In fact, these two sets of equations imply, and are implied by, each other. In conclusion, the dual set of jump conditions associated with mass, boosts and expansions, is completely equivalent to the usual Rankine-Hugoniot conditions and may be used for an independent description of the shock.

\section{The Entropy Condition}

For a polytropic gas, by choosing $\varepsilon=\chi \rho^{\gamma_{0}}$, we can rewrite Eq. (5) as

$$
D \chi=0
$$

In 5] we defined an isentropic flow to be one for which $\chi=$ constant. For a general flow, it followed that $\chi$ transforms like a scalar. For a polytropic gas, it is also well-known [8] that $\chi$ is related to the specific entropy (entropy per unit mass), $S$ as follows:

$$
S-S_{0}=C_{v} \log \left[\chi(\rho V)^{\gamma_{0}}\right]
$$

where $C_{v}=R /\left(\gamma_{0}-1\right), R$ being the universal gas constant divided by the molecular weight of the particular gas, $V$ the volume and $S_{0}$ an appropriate constant. It is obvious from this equation that as a particle of the medium moves about, the specific entropy at the moving particle remains constant under an $S L(2, R)$ transformation. Hence, under an $S L(2, R)$ transformation, a physical shock gets mapped to a physical shock.

We now require the positivity of viscosity to be preserved under an $S L(2, R)$ transformation - a requirement that guarantees that the shock respects the 
entropy condition. As already pointed out (see eq. (12)), in three-dimensional space, the viscosity fields transform as follows:

$$
\eta^{\prime}=(\gamma t+\delta)^{3} \eta \quad \text { and } \quad \zeta^{\prime}=(\gamma t+\delta)^{3} \zeta
$$

Thus the transformation properties of the viscosity fields are similar to $\rho$ i.e., they transform like scalar densities. Hence, if we integrate the viscosity field over all space, to get the viscosity, it is an invariant under the $S L(2, R)$ transformations. Likewise, the specific viscosity (viscosity per unit mass), is an invariant. It follows that the positivity of the viscosity is maintained without any additional restrictions on the $S L(2, R)$ parameters.

\section{Conclusions}

In this paper, we extended the analysis of [5] to discuss the symmetries of discontinuous flows in fluid dynamics. The maximal kinematical invariance group of an ideal, polytropic fluid is $\mathcal{G}=S L(2, R) \wedge G$, not just for smooth, but for discontinuous flows also. This is made manifest by writing the fluid equations in their conservation law form. New conservation laws follow from a direct application of Noether's theorem, enabling us to construct a dual set of RankineHugoniot shock conditions. The $S L(2, R)$ transformations map the standard Rankine-Hugoniot shock conditions to the dual ones and vice versa. These transformations also respect the entropy conditions that physical shocks need to satisfy. Hence we conclude that, under these transformations, an explosion gets mapped to an implosion, thus offering a theoretical explanation for the intriguing observations of [1, 2, 3].

\section{Acknowledgements}

We thank L. O'C Drury, Pravir Dutt and S.G. Rajeev for discussions. VVS thanks A. J. Niemi for his hospitality in Uppsala, Sweden, and the Center for Dynamical Processes and Structure Formation (CDP), Uppsala University, for financial support.

\section{References}

[1] B. Remington, "Supernova Hydrodynamics Up Close," Science and Technology Review Jan'/Feb' 2000, Lawrence Livermore National Laboratory; http://www.llnl.gov/str

[2] I. Hachisu et al., "Rayleigh-Taylor instabilities and mixing in the helium star models for Type Ib/Ic supernovae," Astrophysical Journal 368 (1991) L27-30; 
[3] H. Sakagami and K. Nishihara, "Rayleigh-Taylor instability on the pusherfuel contact surface of stagnating targets," Physics of Fluids B 2 (1990) $2715-2730$.

[4] L. O'C Drury and J. T. Medonça, "Explosion Implosion Duality and the Laboratory Simulation of Astrophysical Systems", Physics of Plasma 7 (2000) 5148-5152

[5] L. O'Raifeartaigh and V. V. Sreedhar, "The maximal kinematical invariance group of fluid dynamics and explosion-implosion duality," Annals Phys.293:215-227,2001 hep-th/0007199

[6] In a different context, this group was first discovered by R. Jackiw, Phys. Today 25 (1972) 23; U. Niederer, Helvetica Physica Acta, 45 (1972) 802; C. R. Hagen, Phys. Rev D5 (1972) 377. For related work in fluid mechanics see, M. Hassaine and P. A. Horvathy, Ann. of Phys. 282 (2000) 218; A. M. Grundland and L.Lalague, Can. J. Phy. 72 (1994) 362, Can. J. Phys. 73, (1995) 463. For a review see R. Jackiw, physics/0010042.

[7] Robert D. Richtmyer, Principles of Advanced Mathematical Physics, Vol. 1 1978 Springer-Verlag, See section 2.9, $17.1-17.6$

[8] R. Courant and K. O. Friedrichs, Supersonic Flow and Shock Waves, 1948 Interscience Publishers, Inc., New York.

[9] L. D. Landau and E. M. Lifshitz, Fluid Mechanics, Pergamon Press (1959).

[10] Herbert Goldstein, Classical Mechanics, Second Edition 1980, Addison Wesley, See section 12-7.

[11] O. Jahn and V. V. Sreedhar, Am. J. Phys. 69, 1039 (2001) math-ph/0102011 